\title{
Regge theory and statistical mechanics
}

\author{
F. Canfora, L. Parisi, G. Vilasi \\ Istituto Nazionale di Fisica Nucleare, Sezione di Napoli, GC di Salerno \\ Dipartimento di Fisica "E.R.Caianiello", Università di Salerno \\ Via S.Allende, 84081 Baronissi (Salerno), Italy
}

\begin{abstract}
An interesting connection between the Regge theory of scattering, the Veneziano amplitude, the Lee-Yang theorems in statistical mechanics and nonextensive Renyi entropy is addressed. In this scheme the standard entropy and the Renyi entropy appear to be different limits of a unique mathematical object. This framework sheds light on the physical origin of nonextensivity. A non trivial application to spin glass theory is shortly outlined.
\end{abstract}

Key words: Nonextensive entropy, Regge theory, Veneziano amplitude.

PACS: : 12.40.Nn,11.55.Jy, 05.20.-y, 05.70.-a.

\section{Introduction}

Quantum information theory is one of the hottest topic in physics because of its theoretical and phenomenological interest. One of the main tools needed to analyze the open problems in this fascinating field is the concept of entropy which, by the way, plays a key role in all theoretical as well as experimental fields. The standard point of view, which is able to describe a huge amount of observations, is the standard $B G N S^{1}$ entropy which is

$$
S\left(\left\{p_{i}\right\}\right)=-\sum_{i} p_{i} \ln p_{i}
$$

where $\left\{p_{i}\right\}$ is a distribution of probability

$$
\begin{aligned}
\left\{p_{i}\right\} \quad i=1, \ldots, N \mid \quad p_{i} & \geq 0 \quad \forall i, \\
\sum_{i} p_{i} & =1 .
\end{aligned}
$$

$\overline{1 B G N S}$ stands for Boltzmann-Gibbs-Von Neumann-Shannon. 
From the above definition, the equilibrium thermodynamics (both classical and quantum) follows, as usual, by using well known constrained minimizations procedures. On the other hand, there is an increasing amount of data indicating that, in certain circumstances (such as multiparticles hadronic systems, fractional diffusion processes, multifractal systems, coding theory and cryptography, chaotic dynamical systems, quantum-informations uncertainty relations and so on; for an updated list of references see [18]) a different notion of entropy is more suitable. In the above mentioned cases the nonextensive Renyi entropy [18] appears to be the right one.

Given a distribution of probability as in Eqs. (1) and (2), the Renyi entropy $S_{R}^{b}$ of index $b$ is defined as

$$
S_{R}^{b}\left(\left\{p_{i}\right\}\right)=\log _{2}\left[\left(\sum_{i} p_{i}^{b}\right)^{\frac{1}{1-b}}\right]
$$

It reduces to the $B G N S$ entropy when $b \rightarrow 1$.

Besides the Renyi entropy, also the nonextensive Tsallis entropy [21] is able to describe many phenomena (such as systems with long-range interactions) in which the standard extensive entropy seems to fail. For these reasons it would be important to shed more light on the conceptual relations between these different notions of entropy. In particular, it will be shown that the fact that the Renyi entropy (as well as the Tsallis entropy) approaches the standard one when a suitable parameter goes to 1 is not at all the end of the story. One of the main problems affecting these two notions of entropy is the following: even if many phenomena are very well described by nonextensive entropies, the index of nonextensivity changes from phenomenon to phenomenon. It is not clear the physical information hidden in the nonextensivity index and, therefore, it is not clear the mechanism which leads to such a "non universality". The main scope of the paper is to propose an interpretation of the nonextensivity index able to shed light on these questions.

Indeed, there is a very rich analytical structure connecting these different entropies which could provide many phenomena with a new understanding (such as the meaning of the parameter $b$ present in the Renyi entropy of which, at a present time, it is available only a phenomenological interpretation as a parameter measuring the nonextensivity).

Quite surprisingly, the mathematical framework which underlies all these entropies is the Regge theory of scattering $[16,17]$ when applied to tree diagrams in string theory. To begin with, let us recall the expression of the Veneziano amplitude [22] $A_{V}$ 


$$
\begin{aligned}
A_{V}(t, s, u)= & c_{1}\left[B\left(-\alpha_{0}(s),-\alpha_{0}(t)\right)+B\left(-\alpha_{0}(s),-\alpha_{0}(u)\right)\right. \\
& \left.+B\left(-\alpha_{0}(t),-\alpha_{0}(u)\right)\right] \\
B\left(-\alpha_{0}(x),-\alpha_{0}(y)\right)= & \frac{\Gamma\left(-\alpha^{\prime} x-1\right) \Gamma\left(-\alpha^{\prime} y-1\right)}{\Gamma\left(-\alpha^{\prime} x-\alpha^{\prime} y-2\right)} \\
s+t+u= & N, \quad s, t, u \geq 0
\end{aligned}
$$

where $B$ is the Euler beta function, $s, t$ and $u$ are the Mandelstam variables, $\Gamma$ is the Euler gamma function and $c_{1}, \alpha^{\prime}$ and $N$ are constants. The beautiful analytical structure of the Veneziano amplitude, which is based on the Regge theory of scattering, describes quite well certain features of strong interactions and, eventually, it has been recognized his string-theoretical origin: it can be derived by computing the vacuum expectation value of four tachyonic operators on the disk. As far as the above themes are concerned, two limits are interesting: the Regge limit and the hard scattering limit.

The Regge limit is

$$
\begin{aligned}
|s| & \rightarrow \infty, \quad t \quad \text { fixed } \\
A_{V} & \approx c_{1} s^{\alpha_{0}(t)} \Gamma\left(-\alpha_{0}(t)\right) \approx c_{1} \exp \left[\ln \left(s^{\alpha_{0}(t)}\right)\right] \Gamma\left(-\alpha_{0}(t)\right), \\
\alpha_{0}(t) & =\alpha^{\prime} t+1
\end{aligned}
$$

the above Regge behavior is valid in all the complex $s$-plane as long as $s$ is far away the real axis.

The hard scattering limit is

$$
\begin{aligned}
&|s| \rightarrow \infty, \quad t / s \quad \text { fixed } \\
& A_{V} \approx c_{1} \exp \left[-\alpha^{\prime}(t \ln t+s \ln s+u \ln u)\right] .
\end{aligned}
$$

It is convenient to rescale the Mandelstam variables as follows

$$
s=p_{1} N, \quad t=p_{2} N, \quad u=p_{3} N .
$$

so that

$$
p_{1}+p_{2}+p_{3}=1 \text {. }
$$

In terms of the rescaled variable, the Regge and the hard scattering limits of the Veneziano amplitude respectively read

$$
\begin{array}{rl} 
& \left(N \rightarrow \infty,\left|p_{2}\right| /\left|p_{1}\right|,\left|p_{3}\right| /\left|p_{1}\right| \quad \text { small }\right) \\
A_{V} \approx & c_{1} \exp \left[-c_{2} S_{R}^{b}\left(\left\{p_{i}\right\}\right)+c_{3}\right] \\
& \left(N \rightarrow \infty,\left|p_{2}\right| /\left|p_{1}\right| \quad \text { fixed }\right) \\
A_{V} \approx & c_{4} \exp \left[-N \alpha^{\prime} S\left(\left\{p_{i}\right\}\right)+c_{5}\right] \\
c_{1}, c_{2}, c_{4}>0 & 0
\end{array}
$$


where $c_{2}, c_{3}, c_{4}$ and $c_{5}$ are constants and the index $i$ goes from 1 to 3 . In Eqs. (9) and (10) it has been taken into account that $p_{2}$ and $p_{3}$ are small with respect to $p_{1}$ and the index of the Renyi entropy is determined by

$$
\frac{b}{1-b}=\alpha_{0}(t) \approx 1+o\left(p_{2}\right) \Rightarrow b \approx \frac{1}{2}+o\left(p_{2}\right)
$$

where, in this context, it is natural to interpret the constant $N$ as a measure of the number of degrees of freedom of the system and, consequently, the limit $N \rightarrow \infty$ as a thermodynamical limit. Thus, we have obtained the interesting result that the complex exponential of (minus) the Renyi entropy can be seen (once the probabilities $p_{i}$ are promoted to complex variables) as a "Regge-like" limit of the exponential of the Veneziano amplitude. In other words, (one over) the partition function (one gets $1 / Z$ because in the exponent it appears minus the entropy) of a system characterized by a Renyi entropy can be seen as the "Regge-limit" of (one over) the partition function of a system characterized by the standard $B G N S$ entropy in the hard scattering limit. This analysis also shows that the index of nonextensivity $b$ of the Renyi entropy is related to the probabilities which, for some reasons, become negligible during the evolution. To be more specific, the analytic continuation of the exponential of minus the standard entropy to complex values of the probabilities $p_{i}$ in the Regge limit gives the (complex version of the) exponential of minus the Renyi entropy with a nonextensive parameter which is connected to the small probabilities. In this picture, the nonextensivity seems to be related to states which, in principles, are available but at which, for dynamical reasons, the probabilities to arrive is negligible. One can look at this property also from a different perspective: the "statistical" hard scattering limit is completely symmetric in the probabilities so that crossing symmetry (which, in this statistical framework, is nothing but the invariance of the standard entropy under a permutation of the $p_{i}$ ) is explicit. On the contrary, the "statistical" Regge limit is not symmetric with respect to the probabilities (since some are negligible with respect to others): a breaking of the crossing symmetry leads, upon analytic continuation, to a nonextensive entropy in which there is a reduced invariance of the entropy under separate permutations of the large probabilities and of the small probabilities. Thus, in a sense, the cases in which, for dynamical reasons, the states (and, consequently, the related probabilities) are not equivalent ${ }^{2}$, nonextensive entropy should come into play (the present conclusion is supported by some already known results in the literature: see, for instance, [6]).

It is worth to note here that, because of the power of holomorphy, the requirement of crossing symmetry of the Veneziano amplitude was (together with the Regge theory) crucial in deriving the explicit expression (4) and its hard scattering and Regge limits. Thus, the high energy physics analogy sug-

2 This could happen, for example, when the phase space is made of disconnected "islands" which, therefore, cannot be compared with continuous transformations. 
gests that quite in general, once one promotes the probabilities to complex variables, the analytic continuation of the exponential of minus the standard entropy very likely is the (complex) exponential of minus the Renyi entropy (in which the complex probabilities are the ones with the largest moduli). Also the interpretation of the Regge poles is quite interesting. The Regge poles of the Veneziano model are located at

$$
s, t=\alpha^{\prime}(n-1)
$$

so that

$$
\left|p_{i}\right|=\alpha^{\prime} \frac{n_{i}-1}{N}
$$

where $n_{i}$ is an integer. Consequently, the analytic continuation of the exponential of the standard entropy should have poles for negative probabilities (away from the "physical" real axis) when the moduli of (some of) the probabilities have a rational values (setting $\alpha^{\prime}=1$ ).

The beautiful results of Lee and Yang $[12,13]$ are very useful in clarifying the physical meaning of the above analogy between Regge scattering theory and statistical mechanics. Lee and Yang argued that if one promotes the inverse temperature $\beta$ and the magnetic field $h$ to be complex variables (as well as other couplings with external fields which, in case, could be present) the zeros of the partition function in the complex $\beta, h$ plane lie on lines in the complex planes far away from the real axis. The number of zeros increases with the size of the system and, in the thermodynamic limit they should form brunch cuts starting from the real axis. Hence, from the point of view of complex analysis in the complex $\beta, h$ plane, phase transitions are related to pinching phenomena which prevent the analytic continuation ${ }^{3}$. It is then quite natural, instead to promote $\beta$ and $h$ to complex variables while keeping real the probabilities, to promote the probabilities (and, in case, other local fields) to be complex variables while keeping real the external parameters. In this case, the results of Lee and Yang can be restated by saying that the singularities of the free energy lie on lines in the complex probabilities plane which, in the thermodynamic limit, tend to form brunch cut emanating from the real axis. This is precisely what Eqs. (13) and (14) say: in the thermodynamic limit $N \rightarrow \infty$ the poles of the Veneziano amplitude (which, as it has been already remarked, is the inverse of the analytic continuation of a standard partition function) cumulate forming brunch cut(s) emanating from the physical real axis.

The formal generalization of this relation in the cases in which the vector of probabilities is $N$-dimensional, is a relatively straightforward computation in string theory in which, instead of computing the vacuum expectation values on the disk of four tachyonic operators, one considers the case of $N+1$

3 The Lee-Yang results have been tested in many situations, however a rigorous proof in the general case is not available yet. 
tachyonic operators. However, it is worth to stress that the generalization of the Veneziano amplitude was obtained before the emergence of its relation with string theory (see, for example, [5]). In (the exponential of) the standard entropy

$$
\exp \left[-\alpha \sum_{i}^{N} p_{i} \ln p_{i}\right],
$$

the probabilities can be thought as complex variables and the above exponential can be analytically continued in the spirit of Eq. (8) in which one identifies probabilities with rescaled Mandelstam variables. The result is a generalized Veneziano amplitude which, in the hard scattering limit, gives back the (exponential of minus the) standard entropy and in the Regge limit gives the Renyi behavior (a detailed description of the analytic structure of the generalized Veneziano amplitude can be found in [7]; an analysis of the mathematical properties of the generalized Veneziano amplitude in a modern perspective can be found in [10]). For instance, one can choose a $k$-dimensional subset $\left\{p_{1}, . ., p_{k}\right\}$ of the probabilities $\left\{p_{i}\right\}$ with respect to which to consider the Regge limit ${ }^{4}$ :

$$
N \rightarrow \infty, \quad\left\{p_{1}, . ., p_{k}, p_{k+1}, . ., p_{n}\right\} \mid \quad p_{l} \ll p_{m} \quad \forall l \leq k, \forall m>k .
$$

In this case the Renyi behavior manifests itself as follows

$$
\exp [-\alpha S(k, n-k)] \approx \exp \left[-c_{6} S_{R}^{b}\left(P_{i-k}\right)+c_{7}\right]
$$

where the notation $S(k, n-k)$ stresses the fact that one is taking the Regge limit with respect to the first $k$ probabilities, $P_{i-k}$ is the total probability to be in one of the "non negligible states" (that is, the states which have non negligible probabilities in the Regge limit), $c_{6}$ and $c_{7}$ are suitable constants and the nonextensivity parameter $b$ is connected to the negligible probabilities

$$
b=b\left(\alpha_{0}\left(p_{i_{k}}\right)\right)
$$

It is interesting to note that, as long as none of the probabilities is negligible with respct to the others, the entropy keeps its standard form while, when some probabilities are negligible, the nonextensive behavior manifests itself. The power of holomorphy together with the crossing symmetry strongly constraint the expected singularities in the complex probabilities plane. The above observations together with Regge theory could be useful to extend the Lee-Yang theorems.

The above method opens the possibility to study the analytic continuation from standard to Renyi entropy in the spirit of the Lee-Yang theorems $[12,13]$.

4 Indeed, it is always possible to arrange the probability vector in such a way that the probabilities with respect to which one takes the Regge limit (which are negligible compared to the other probabilities) appear in the first $k$ components. 
For instance, in the mean field approach to spin-glass systems, the replica method works in the cases in which, at high enough temperatures, one can continue a suitable nonextensive free-energy (with respect to the nonextensive parameter) to obtain the standard free energy. However, the remarkable discovery of Parisi ${ }^{5}$ [15] has been that, at low temperatures the procedure does not work (this is the so-called "replica symmetry breaking") because of the presence of many metastable states (only recently $[8,9,19]$ it has been possible to prove rigorously that the Parisi solution is the right one to describe the mean field glassy phase). A very important quantity in spin glass theory (to provide an updated list of references is an hopeless task; for two detailed review, see $[14,2]$ ) is the TAP free energy (introduced in [20]) $f_{T A P}$

$$
\begin{aligned}
f_{T A P}= & -\frac{1}{2} \sum_{i \neq j} J_{i j} m_{i} m_{j}-\sum_{i} h_{i} m_{i}-\frac{\beta}{4} \sum_{i \neq j} J_{i j}\left(1-m_{i}^{2}\right)\left(1-m_{j}^{2}\right)+ \\
& +\frac{1}{2 \beta} \sum_{i}\left[\left(1+m_{i}\right) \log \left(\frac{1+m_{i}}{2}\right)+\right. \\
& \left.+\left(1-m_{i}\right) \log \left(\frac{1-m_{i}}{2}\right)\right]
\end{aligned}
$$

where $m_{i}$ is the mean value of the magnetization on the site $i, J_{i j}$ are the fluctuating spin couplings (it is usually assumed that they are Gaussian variables with the same mean and variance). In the framework of mean field theory, the TAP free energy provides a spin glass system above the glass transition with a detailed description; the TAP free energy $f_{T A P}$, to work also below the transition, needs more refined arguments [14]. It is interesting to promote, as in the previous case, the variables $m_{i}$ to complex variables and to try to continue ${ }^{6}$ the exponential of $f_{T A P}$. Since the first tree terms in $f_{T A P}$ are polynomials, their analytic continuation does not present problems. It is the last term, related to the (standard $B G N S$ ) entropy, which generically has a non trivial analytic continuation. In the high temperature phase, crossing symmetry in the variables $p_{i}$ defined below, is a good symmetry, so that previous arguments based on the Veneziano amplitude tell that the analytic continuation of the entropic term should give rise to a new entropic term in which, in fact, the Renyi entropy appears. Thus, one can expect that

5 Not to be confused with one of the authors.

6 It is worth to note here that typical tools of high energy physics, such as the BRST formalism and supersymmetry, already appeared in spin glass theory (see, for example, [1] and references therein). 


$$
\begin{aligned}
f_{T A P}^{a c} \sim & -\frac{1}{2} \sum_{i \neq j} J_{i j} m_{i} m_{j}-\sum_{i} h_{i} m_{i}-\frac{\beta}{4} \sum_{i \neq j} J_{i j}\left(1-m_{i}^{2}\right)\left(1-m_{j}^{2}\right)+ \\
& +\frac{\alpha}{2 \beta} \log _{2}\left[\frac{1}{N}\left(\sum_{i}\left(p_{i}\right)^{b}+\left(\bar{p}_{i}\right)^{b}\right)^{\frac{1}{1-b}}\right] \\
p_{i}= & \frac{1+m_{i}}{2 N}, \quad \bar{p}_{i}=\frac{1-m_{i}}{2 N}, \quad i, j=1, . ., N
\end{aligned}
$$

where $b$ and $\alpha$ are suitable constants and the last term in the right hand side of Eq. (16) is the Reny-like term. A comparison with known results in spin glass theory the [11] [4] [3] shows that the interpretation of the standard $B G N S$ entropic term in the TAP free energy as the hard scattering limit of a suitable string theoretical amplitude gives, in the Regge limit, an expression which bears a strong resemblance with the low temperature TAP free energy suitable to study the glassy phase. Thus, the hard scattering and Regge limits correspond to unbroken replica phase and replica symmetry breaking respectively. The non trivial benefit of this method is that, besides to clarify the physical origin of "nonextensivity", it could be easy to handle because of the huge amount of works (in which one could find explicit computations important for statistical mechanics) already available in string theory.

\section{References}

[1] A. Annibale, A. Cavagna, I. Giardina, G. Parisi, E. Trevigne, "The role of the Becchi-Rouet-Stora-Tyutin supersymmetry in the calculation of the complexity for the Sherrington-Kirkpatrick model" J. Phys. A 36, 10937 (2003).

[2] T. Castellani, A. Cavagna, "Spin-Glass Theory for Pedestrians" J. Stat. Mech. (2005) P05012 and references therein.

[3] A. Crisanti, H. J. Sommers "Thouless-Anderson-Palmer Approach to the Spherical p-Spin Spin Glass Model" J. Physique I 5, 805 (1995).

[4] L. F. Cugliandolo and J. Kurchan, "Analytical solution of the off-equilibrium dynamics of a long-range spin-glass model" Phys. Rev. Lett. 71, 173 (1993).

[5] K. Bardakci, H. Ruegg, "Reggeized resonance model for the production amplitude", Phys. Lett. B 28, 5, 342 (1968); M. Virasoro, "Generalization of Veneziano's Formula for the Five-Point Function", Phys. Rev. Lett. 22, 1, 37 (1969); H.M. Chan, "A generalised Veneziano model for the N-point function", Phys. Lett. B 28, 6, 425 (1969); H.M. Chan, S.T. Tsou, "Explicit construction of the N-point function in the generalized Veneziano model", Phys. Lett. B 28, 7, 485 (1969); C.J. Goebel, B. Sakita, "Extension of the Veneziano Form to N-Particle Amplitudes", Phys. Rev. Lett. 22, 6, 257 (1969). 
[6] H.-T. Elze, T. Kodama, "On supercorrelated systems and phase space entrainment" Phys.Lett. A335 (2005) 363; "Dynamical Correlations as Origin of Nonextensive Entropy" Europhysics Letters 70, 439 (2005).

[7] S. Fubini, G. Veneziano, "Level Structure of Dual-resonance Models" Nuovo Cimento 64 A, 811 (1969).

[8] F. Guerra, "Broken Replica Symmetry Bounds in the Mean Field Spin Glass Model" Comm. Math. Phys. 233, 1 (2003).

[9] F. Guerra, F. L. Toninelli, "Central limit theorem for fluctuations in the high temperature region of the Sherrington-Kirkpatrick spin glass model" J. Math. Phys. 43, 6224 (2002); "The Thermodynamic Limit in Mean Field Spin Glass Models" Comm. Math. Phys. 230 (2002), 71.

[10] Arkady L. Kholodenko, "New strings for old Veneziano amplitudes II. Group-theoretic treatment" hep-th/0411241, J.Geom.Phys.(in press); "New Strings for Old Veneziano Amplitudes I.Analytical Treatment" J.Geom.Phys. 55 (2005) 50; "New Strings for Old Veneziano Amplitudes III. Symplectic Treatment" hep-th/0502231, J.Geom.Phys.(in press); "New Models for Veneziano Amplitudes: Combinatorial, Symplectic and Supersymmetric Aspects" Int.J.Geom.Meth.Mod.Phys. 2 (2005) 563.

[11] J. Kurchan, G. Parisi, M.A. Virasoro "Barriers and metastable states as saddle points in the replica approach", J. Physique I 3, 8, 1819 (1993).

[12] C. N. Yang, T. D. Lee, "Statistical Theory of Equations of State and Phase Transitions. I. Theory of Condensation" Phys. Rev. 87, 404 (1952).

[13] C. N. Yang, T. D. Lee, "Statistical Theory of Equations of State and Phase Transitions. II. Lattice Gas and Ising Model" Phys. Rev. 87, 410 (1952).

[14] M. Mezard, G. Parisi, M. Virasoro "Spin Glass Theory and Beyond" World Scientific, Singapore (1987).

[15] G. Parisi, "Infinite Number of Order Parameters for Spin-Glasses", Phys. Rev. Lett. 43, 1754 (1979); " Toward a mean field theory for spin glasses", Phys. Lett. A 73, 3, 203 (1979); "A sequence of approximated solutions to the $S$-K model for spin glasses", J. Phys. A 13, 4, L115 (1980); "The order parameter for spin glasses: a function on the interval 0-1", J. Phys. A 13, 3, 1101 (1980); "Magnetic properties of spin glasses in a new mean field theory", J. Phys. A 13, 5, 1887 (1980); "Order Parameter for Spin-Glasses" Phys. Rev. Lett. 50, 1946 (1983).

[16] T. Regge, "Introduction to Complex Orbital Momenta" Nuovo Cimento 14, 951 (1959).

[17] T. Regge, "Bound States, Shadow States and Mandelstam Representation" Nuovo Cimento 18, 947 (1960).

[18] A. Rènyi, "On Measures of Entropy and Information." J. Neyman, editor, Proceedings of the 4th Berkley Conference on Mathematical Statistics and 
Probability, 547-561, Berkley (1961), University of California Press; see, for an updated list of references on nonextensive statistical mechanics, http://tsallis.cat.cbpf.br/biblio.htm.

[19] M. Talagrand, "The Generalized Parisi Formula" C. R. Acad. Sci. Paris, Series I 337, (2003) 111; " The Parisi Formula" Annals of Mathematics 163, 221-263 (2006).

[20] J. D. Thouless, P. W. Anderson, R. G. Palmer, "Solution of Solvable model of a spin glass" Phil. Mag. 35, 593 (1977).

[21] C. Tsallis, "Possible generalization of Boltzmann-Gibbs statistics" Jour. Stat. Phys. 52, 479 (1988).

[22] G. Veneziano, "Construction of a Crossing-Simmetric, Regge-Behaved Amplitude for Linearly Rising trajectories" Nuovo Cimento 57 A, 190 (1968). 